\title{
Performance of Turbo Product Codes on the Multiple-Access Relay Channel with Relatively Poor Source-Relay Links
}

\author{
Yizhi Yin, Ramesh Pyndiah and Karine Amis \\ Institut Telecom / Telecom Bretagne, Lab-STICC UMR CNRS 3192 \\ Université Européenne de Bretagne, France \\ Email: \{yizhi.yin, ramesh.pyndiah, karine.amis\}@telecom-bretagne.eu
}

\begin{abstract}
In this paper, we study a cooperative coding scheme based on turbo product codes where a number of sensors transmit to a same destination with the help of a relay. This network can be modeled by a multiple-access relay channel (MARC). In the proposed scheme, the relay applies algebraic systematic Network Coding to the source codewords and forwards only the additional redundancy to the destination where an overall product codeword is observed. Based on the single-relay scenario that has been studied in a previous paper, we analyze the error probabilities at the relay input and output for different relay strategies. Taking into account the residual errors at the relay, an appropriate loglikelihood ratio is used at the destination by the turbo decoder. The error performance under the degraded source-relay channel condition is shown on the Rayleigh fading channel. Besides that, we analyze the error correlation in the relay-generated redundancy and investigate the benefits of using multi-relay cooperation. Different cooperation schemes are compared in terms of performance, complexity and energy consumption.
\end{abstract}

Index Terms-Turbo product codes, cooperative communications, network coding, Rayleigh channel, sensor network.

\section{INTRODUCTION}

We consider the wireless sensor network where many sensors transmit data to a single destination with the help of a relay. These sensors have low complexity and limited energy. Each sensor transmits at a low data rate but the network sum-rate is high thanks to the large number of sensors. The relay with higher computing and energy capabilities is located close to these sensors to improve the transmission. This network can be modeled by a time-division multiple-access relay channel (MARC) which has been studied in [1-3].

Since the introduction of the relay channel model by Cover and El. Gamal [4], cooperative communication using relay techniques has been extensively studied to improve the transmission quality. Through cooperation, we can construct more efficient systems based on different schemes [5] such as the Amplify-and-Forward and the Decode-and-Forward. Recently, Network Coding [6,7] has also attracted much attention. In Network Coding, the transmitted blocks are combined at intermediate nodes and the resulting blocks are forwarded to the destination. In wired error-free networks, one can thus optimize the system throughput and economize the transmission energy. When we use Network Coding in wireless networks,

This work has been financially supported by Région Bretagne and Pracom. one of the main obstacles is the error propagation resulting from the higher error probability in the wireless environment.

The cooperative scheme studied in this paper considers the algebraic Network Coding in the MARC model with timedivided channels to avoid interference. All sources use the same systematic block code and broadcast the codewords to the relay and the destination. Instead of forwarding the received source codewords to the destination, the relay stores all source codewords in the rows of a matrix and encodes the columns using another systematic block code and forwards only the relay-generated redundancy to the destination. At the destination, the codewords from the sources and the redundancy from the relay are decoded iteratively using the turbo product code decoding [8]. Since the source-relay channel is noisy, there are residual errors in the source-relay detected codewords. After relay encoding, these errors are propagated to the relay-generated redundancy, which will degrade the error correction capability at the destination.

Similar cooperative schemes based on turbo product codes have been studied in other papers. For example, [9] investigates the multi-relay cooperation on the Gaussian channel. The error performance is analyzed with different relay positions, but the error propagation is not emphasized. [10] considers the single relay case with an error-free source-relay channel. [11] studies the multi-relay case with the product code based on the Bose-Chaudhuri-Hocquenghem $(\mathrm{BCH})$ codes. In order to mitigate the error propagation, each relay applies a different cyclic interleaving on its detected source $\mathrm{BCH}$ codewords to generate different relay redundancy through column encoding. Each relay sends its whole redundancy to the destination. The error propagation is alleviated at the cost of a reduced data rate and a much higher complexity in the turbo decoding.

In [12], a single relay is considered and $\mathrm{BCH}$ codes are used at both the sources and the relay for the cooperative scheme described above. The turbo decoder considers the residual errors and computes an appropriate log-likelihood ratio (LLR) for the observations coming from the relay-destination channel to alleviate the performance degradation due to the error propagation. High coding gain has thus been obtained. The main advantage of this cooperative scheme is that the turbo code performance can be achieved for each source through the cooperation without increasing the transmission delay usually associated with the turbo-coding at each source. 
This paper extends the single-relay cooperative scheme of [12] and its main contributions are:

1. Investigate the analytical error probabilities at the relay for the hard detection and hard/soft decoding strategies and simulate the corresponding error performance on the fast Rayleigh fading channel with noisy source-relay links.

2. Propose an optimized multi-relay solution to further mitigate the error propagation besides the LLR limitation used in [12]. The turbo decoding complexity and the data rate are not sacrificed contrary to [11].

3. Different cooperation schemes are compared based on the constraints of complexity and energy consumption.

The rest of the paper is organized as follows: Section II recalls the network setup and the LLR-limiter proposed in [12]. Section III investigates the analytical error probability at the relay to find the theoretical LLR-limiter threshold on the Rayleigh channel. Then, the network performance is simulated for the single-relay cooperation. Section IV analyzes the error correlation in the relay-generated redundancy and proposes a multi-relay solution. Finally, we make a comparison of different cooperation schemes. Section V concludes the paper and points out future research directions.

\section{Network SETUP AND LLR-LIMITER}

The cooperative network with multiple sources and multiple relays is depicted in Fig. 1. $k^{\prime}$ sources transmit independent data to the same destination with the help of $m$ relays. All sources use the same systematic block code $(n, k)$ of length$n$ and dimension- $k$. The $k^{\prime}$ source codewords received by each relay are stored in the first $k^{\prime}$ rows of a matrix. The relay then encodes the columns using a second systematic block code $\left(n^{\prime}, k^{\prime}\right)$. The relay-generated column redundancy is sent to the destination. The destination observes a product codeword and applies the turbo decoding algorithm [8] to estimate the source data.

All transmissions are scheduled by the time-division multiple-access (TDMA) mode ensuring no multiple-access interference. We suppose perfect synchronization (time, frequency) and there is no inter-symbol interference at the receiver. We denote the average signal to noise ratio of the source-destination (resp. source-relay and relay-destination) channels as $S N R_{s d}$ (resp. $S N R_{s r}$ and $S N R_{r d}$ ).

The sources are sensors with low complexity and limited energy. The relay has more processing power and energy than the sources and is located close to them to improve the transmission, so the source-relay distance $d_{s r}$ is much smaller than the source-destination distance $d_{s d}$. The relays are considered to be located at the same distance to the destination as the sensors. We have $d_{r d}=d_{s d}$ and $S N R_{r d}=S N R_{s d} . S N R_{s r}$ is higher than $S N R_{s d}$ with a difference of $\Delta S N R(\mathrm{~dB})$ :

$$
S N R_{s r}=S N R_{s d}+\Delta S N R
$$

where $\Delta S N R=10 \log _{10}\left(d_{s d} / d_{s r}\right)^{\alpha}$ and $\alpha$ is the path-loss coefficient and is set to 3.5 for a Rayleigh fading.

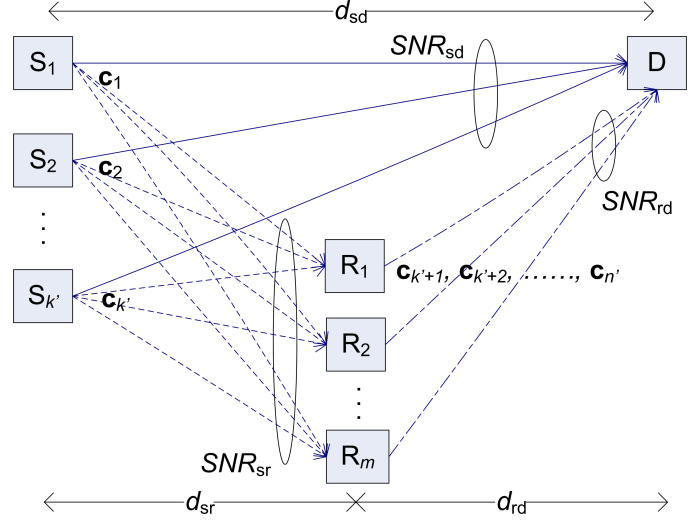

Fig. 1. Multi-source multi-relay sensor network

We denote the $i$-th row of the product codeword generated by the relay without decoding errors at the relay as $\mathbf{c}_{i}=\left(c_{i 1}, c_{i 2}, \ldots, c_{i n}\right), i=1,2, \ldots, n^{\prime}$. For antipodal modulation BPSK, $c_{i j} \in\{ \pm 1\}$. The observation at the destination is:

$$
r_{i l}=\alpha_{i l} \varepsilon_{i l} c_{i l}+b_{i l}
$$

where $\alpha_{i l}$ is the fading coefficient. $b_{i l}$ represents the additive white Gaussian noise (AWGN) with zero mean and variance $\sigma^{2} . \varepsilon$ is a random variable representing the binary error event at the output of the relay encoder with a corresponding bit error probability $\operatorname{Pr}\left\{\varepsilon_{i l}=-1\right\}=p$.

For the source-destination channel, there is no error event at the sources so $p=0$ and the channel output LLR $\lambda_{i l}=\frac{2 \alpha_{i l} r_{i l}}{\sigma^{2}}$. For the observations from the relay-destination channel, it is proved in [12] that for independent residual errors at the relay:

$$
\lambda_{i l} \approx \operatorname{sgn}\left(r_{i l}\right) \cdot \min \left(\frac{2 \alpha_{i l}\left|r_{i l}\right|}{\sigma^{2}},-\ln \frac{p}{1-p}\right) .
$$

We define $x=-\ln \frac{p}{1-p}$ as a limiter threshold on the LLR value at the output of the relay-destination channel. During the turbo decoding, a similar limiter $y$ is applied to the extrinsic information associated with the relay generated redundancy whose value is optimized experimentally through simulations. The system block diagram is depicted in Fig. 2.

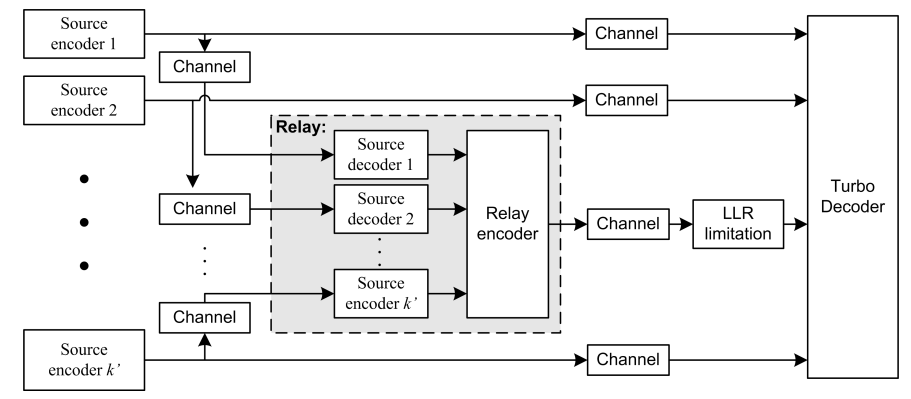

Fig. 2. System block diagram

\section{ERror Performance on RAYleigh ChanNel}

The relay can use different strategies to detect the source codewords such as the hard bit detection, the hard decoding 
and the soft decoding. After estimating the $k^{\prime}$ source codewords, the relay uses the block code $\left(n^{\prime}, k^{\prime}, d_{\text {min }}^{\prime}\right)$ to generate the relay redundancy where $d_{\min }^{\prime}$ is the code minimum Hamming distance. We denote $R$ the code rate and $t^{\prime}$ the code error correction capability. Each column of the product code matrix corresponds to a column codeword generated by relay encoding. Let $\mathbf{m}$ (resp. $\hat{\mathbf{m}}$ ) denote the column message part without (resp. with) errors. We denote $\mathbf{c}$ (resp. $\hat{\mathbf{c}}$ ) the column codeword associated to the message $\mathbf{m}$ (resp. $\hat{\mathbf{m}}$ ). The following considers one column of the matrix.

We denote $p_{i n}$ the bit error probability at the input of relay encoder. For fast Rayleigh fading channel, $p_{i n}$ is given by:

Hard detection : $p_{i n}=p_{e b}^{\star}=\frac{1}{2}\left[1-\sqrt{\frac{R E_{b} / N_{0}}{1+R E_{b} / N_{0}}}\right]$.

Hard decoding:

$$
p_{i n} \leq \frac{2 t^{\prime}+1}{n^{\prime}} \sum_{m=t^{\prime}+1}^{n^{\prime}}\left(\begin{array}{l}
n^{\prime} \\
m
\end{array}\right)\left(p_{e b}^{\star}\right)^{m}\left(1-p_{e b}^{\star}\right)^{n^{\prime}-m} .
$$

Soft decoding [13]:

$$
p_{\text {in }} \leq \sum_{d=d_{\text {min }}^{\prime}}^{n^{\prime}} \frac{d A_{d}}{n^{\prime}}\left[\frac{1-\mu}{2}\right]^{d} \sum_{k=0}^{d-1}\left(\begin{array}{c}
d-1+k \\
k
\end{array}\right)\left[\frac{1+\mu}{2}\right]^{k} \text {. }
$$

where $A_{d}$ is the weight distribution of the column codeword and $\mu=\sqrt{\frac{R E_{b} / N_{0}}{1+R E_{b} / N_{0}}}$. The bit error probability at the relay output (relay-generated redundancy):

$$
p_{e b}^{\text {out }} \leq \sum_{d=d_{m i n}^{\prime}}^{n^{\prime}} \sum_{w=1}^{k^{\prime}} \frac{d-w}{n^{\prime}-k^{\prime}} \operatorname{Pr}\left(\mathrm{d}_{\mathrm{H}}(\mathbf{c}, \hat{\mathbf{c}})=d\right) .
$$

$$
\begin{aligned}
& \operatorname{Pr}\left(\mathrm{d}_{\mathrm{H}}(\mathbf{c}, \hat{\mathbf{c}})=d\right) \\
\leq & \sum_{w=1}^{k^{\prime}} \operatorname{Pr}\left(\mathrm{d}_{\mathrm{H}}(\mathbf{c}, \hat{\mathbf{c}})=d \mid \mathrm{d}_{\mathrm{H}}(\mathbf{m}, \hat{\mathbf{m}})=w\right) \cdot \operatorname{Pr}\left(\mathrm{d}_{\mathrm{H}}(\mathbf{m}, \hat{\mathbf{m}})=w\right) \\
\leq & \sum_{w=1}^{k^{\prime}} \frac{B_{2}(w, d)}{\sum_{s} B_{2}(w, s)} \cdot\left[\left(\begin{array}{c}
k^{\prime} \\
w
\end{array}\right)\left(p_{i n}\right)^{w}\left(1-p_{i n}\right)^{k^{\prime}-w}\right] \cdot
\end{aligned}
$$

where $B_{2}(w, d)$ is the number of column codewords of weight $d$ with message part of weight $w$ and $\mathrm{d}_{\mathrm{H}}(\mathbf{x}, \mathbf{y})$ is the Hamming distance between two vectors.

In this paper, the sources use the $\mathrm{BCH}(64,51)$ code and the relay uses the $\mathrm{BCH}(32,26)$ code. Fig. 3 plots the bit error probability at relay input/output for three relay strategies. The theoretical analysis has been verified by simulations. We use the Chase algorithm [14] for the soft decoding with 128 test patterns to validate the theoretical results. In the following, we use only 16 patterns in the Chase soft decoding at both relay and destination for the sake of complexity reduction.

The difference between the three strategies increases with the SNR. The soft decoding has the lowest error probability at both input and output. The error is amplified at the relay output for each strategy due to the error propagation.

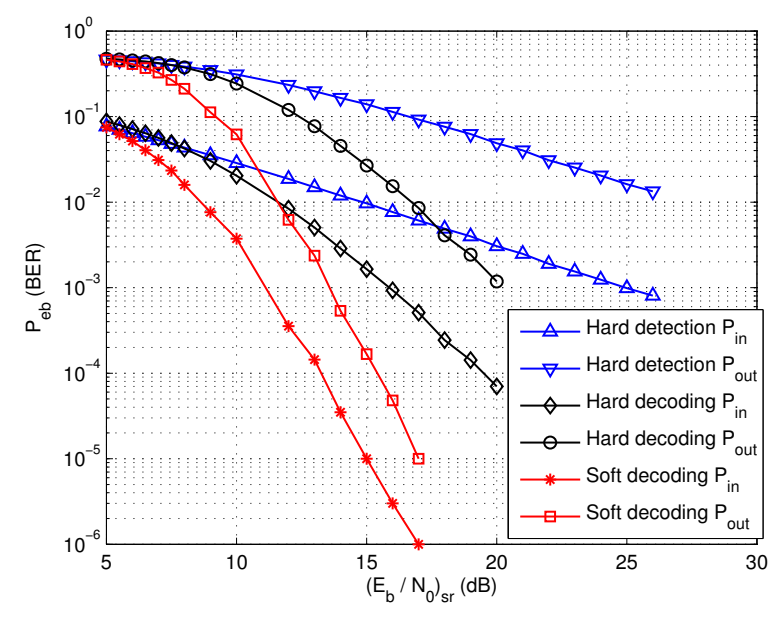

Fig. 3. Bit error probability at relay input/output, fast Rayleigh channel

Fig. 4 plots the BER performance of the single-relay cooperation versus $E_{b} / N_{0}$ for different relay strategies on the fast Rayleigh fading channel. $E_{b}$ is the average received bit energy at the destination including the signal coming from relay. To show the influence of residual errors, we set $\Delta S N R=10 \mathrm{~dB}$ for both the hard-detection and the hard-decoding strategies. For the soft-decoding strategy as there are few residual errors at $\Delta S N R=10 \mathrm{~dB}$, we set $\Delta S N R=8 \mathrm{~dB}$. Theoretically, the LLR limiter $x$ changes with $E_{b} / N_{0}$. To simplify the system, we use a predefined average value for $x$ for all $E_{b} / N_{0}$ considered in Fig. 4 for each strategy, e.g. for the hard detection at relay, we take $E_{b} / N_{0}=8 \mathrm{~dB}$, which is a middle value around which the BER exhibits the error floor. The corresponding $\left(E_{b} / N_{0}\right)_{s r}=18 \mathrm{~dB}$ and we find $x=2.5$ using Fig. 3 and Eq.(3).

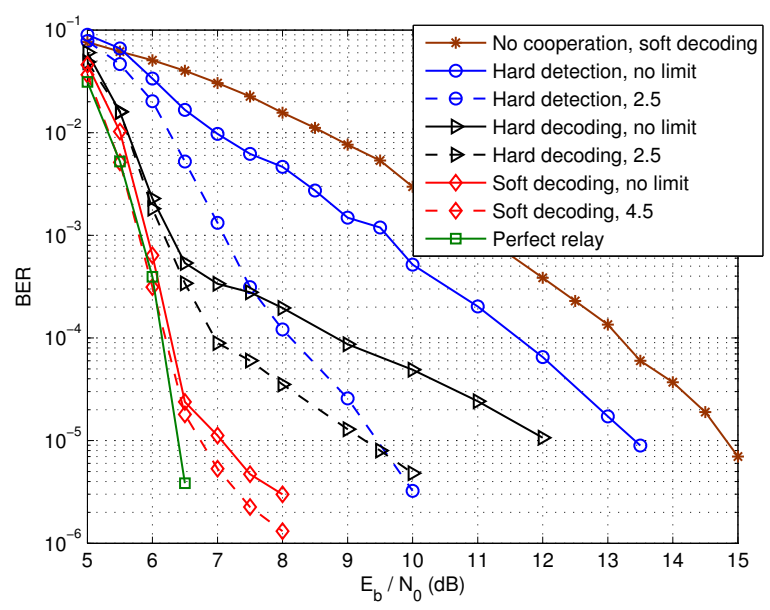

Fig. 4. BER performance of single-relay cooperation, fast Rayleigh fading

In Fig. 4, the curve with stars is the soft-decoding BER of the direct transmission using $\mathrm{BCH}(64,51)$ code without relay. The curve with squares corresponds to the perfect relaying (no residual errors), which is a lower bound for the network BER. 
Using the perfect relay, the coding gain at $\mathrm{BER}=10^{-5}$ is of about $8.4 \mathrm{~dB}$. This gain can be exploited either to extend the transmission range or to reduce the transmission power by a factor of 7 . The other curves correspond to the noisy relaying. The legend indicates the corresponding relay strategy and the average LLR limiter threshold $x$ being used. The limiter $y$ is fixed to 0.5 in all cases. The two curves with circles correspond to the hard-detection strategy case. With the LLR limitation, we get an improvement of about $4 \mathrm{~dB}$ at $\mathrm{BER}=10^{-5}$. We can observe similar improvements for the two other relay strategies. The error performance of the softdecoding strategy is the most robust since it has the smallest residual error numbers after the relay decoding (see Fig. 3).

The errors propagated to the the relay-generated redundancy are correlated and contribute to the error floor in Fig. 4, e.g. for soft-decoding strategy, the relay redundancy sent to the destination corresponds to a product codeword associated with the error pattern at the relay, which is a competitor of the original product codeword.

In simulations, for the hard/soft-decoding strategies, the optimal limiter $x$ is smaller than the theoretical value. It is because Eq.(3) supposes independent residual errors at the relay, which is the case only for the hard-detection strategy. For the hard/soft decoding, the residual errors are correlated.

\section{Multi-Relay CoOperation}

\section{A. Error Correlation and Multi-relay Cooperation}

For the hard-detection strategy, the residual errors are independently distributed in the first $k^{\prime}$ rows of the relay product encoding matrix. The relay encodes every column using the block code $\left(n^{\prime}, k^{\prime}\right)$. The relay-generated parity bits are contaminated by the errors at the relay input and these errors are correlated column-wise. For the soft-decoding strategy, the decoded source codewords are still valid source codewords although they may contain errors, in which case the residual errors are correlated row-wise. After relay encoding and according to the product code property, the residual errors are propagated to the relay redundancy and they are correlated both row-wise and column-wise. The error correlation for the hard-decoding strategy is more complicated.

In order to mitigate the influence of the error correlation, we investigate the use of multiple relays. Fig. 5 shows an example with two relays. Each block represents the relay-generated redundancy. The left block is horizontally divided into two parts and each part corresponds to the data forwarded by one of the relays. By such a row-wise division, the column-wise correlation is reduced, which is suitable for the hard-detection relay strategy where there is no row-wise error correlation.
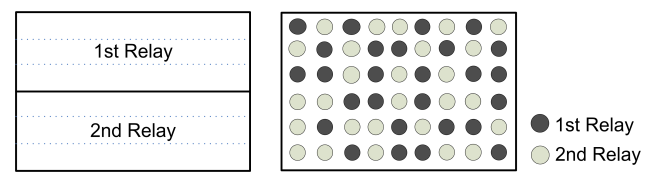

Fig. 5. Row-wise division/pseudo-random selection of relay redundancy
For hard / soft decoding relay strategies, the relay-generated redundancy contains both row-wise and column-wise error correlation. So we propose the pseudo-random (PR) selection of the relay redundancy (right block) where every relay redundancy bit observed by the destination comes from either the first relay or the second relay in a pseudo-random manner. This reduces the error correlation in both directions.

Both methods are multi-relay extensible. Contrary to [11], each relay forwards only a fraction of the whole relay redundancy, so we preserve the turbo decoder complexity and the data rate. If we use more relays, the product codeword observed at the destination will contain less error correlation so that the error floor effect in Fig. 4 can be alleviated. For multirelay cooperation where there is little error correlation, the LLR limiter threshold given in Eq. (3) can be directly applied to all relay strategies and we can easily calculate the error probability at the relay output by using Eq. (7).

Fig. 6 plots the network BER with soft decoding at the relay(s). We set $\triangle S N R=4 \mathrm{~dB}$, which corresponds to a relatively unfavorable condition $\left(d_{s d} \approx 1.3 d_{s r}\right)$. The curve with stars corresponds to the perfect relaying. All the other curves correspond to the noisy relaying combined with the LLR limitation. Here we show the improvements brought by the multi-relay cooperation. The curve with squares is the single-relay case. Using the row-wise division scheme, the 2-relay (curve with circles), 3-relay (curve with triangles) and 6-relay (curve with diamonds) cooperation gradually improves the error performance and alleviates the error floor. With the pseudo-random selection, the performance of 2-relay cooperation outperforms the 3-relay cooperation using the row-wise division.

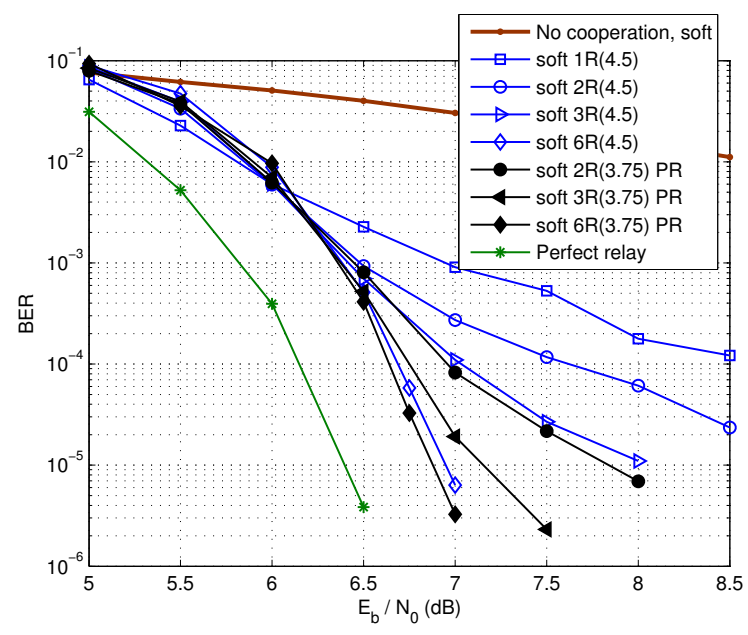

Fig. 6. BER performance with the soft decoding at the relay(s), row-wise division/pseudo-random selection, fast Rayleigh fading channel

The 6-relay cooperation offers the near-best BER and the improvement brought by more relays is negligible. There is a $0.5 \mathrm{~dB}$ difference on $E_{b} / N_{0}$ between the near-best performance and the lower bound of perfect relaying. It is due to the low $\Delta S N R=4 \mathrm{~dB}\left(p_{e b}^{o u t} \approx 2 \times 10^{-2}\right.$ at $E_{b} / N_{0}=7 \mathrm{~dB}$, see Fig. 3$)$.

Similar improvements can also be observed with the hard detection/decoding relay strategies. For hard-detection strat- 
egy, there is no major benefit in using the pseudo-random selection since there is no row-wise error correlation.

\section{B. Comparison of Different Relay Strategies}

For most practical applications in sensor networks, previous work usually considered $d_{s d} \geq 4 d_{s r}(\Delta S N R \geq 21 \mathrm{~dB})$. Here we consider $d_{s d}<4 d_{s r}$ in order to establish the lower limit of the operating range for each relay strategy. In Fig. 7, we compare the network performance for three relay strategies. The abscissa represents $\triangle S N R$ and the ordinate represents the $E_{b} / N_{0}$ necessary to reach a network $\mathrm{BER}=10^{-5}$.

The curves with triangles correspond to the hard-detection strategy. The dashed curves correspond to the hard-decoding strategy. The last three curves in the legend correspond to the soft-decoding strategy. In the figure, all multi-relay cooperation schemes use the row-wise division method.

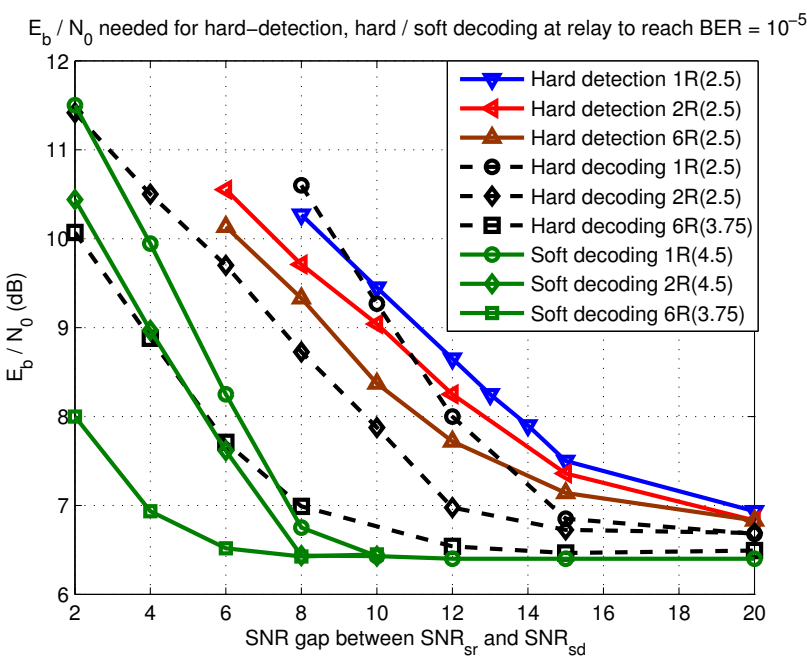

Fig. 7. Comparison of different schemes on fast Rayleigh fading channel

We observe three cases. When $\triangle S N R$ is high enough $\left(\geq 20 \mathrm{~dB}, d_{s d} \geq 3.8 d_{s r}\right)$, the three relay strategies have similar performance. The single-relay cooperation with the harddetection strategy is the best solution as it exhibits the lowest complexity. For middle $\Delta S N R(8 \mathrm{~dB}$ to $20 \mathrm{~dB})$, the single-relay scheme with soft decoding and the multi-relay scheme with hard decoding perform almost the same and there is only a minor degradation at $\Delta S N R=8 \mathrm{~dB}$ for the first one compared to the near-best performance. The multi-relay scheme implies multiple demodulation/decoding of source codewords (each relay receiver has to demodulate and decode them). The additional complexity compared to the single relay case is much higher than that brought by the soft decoding compared to the hard decoding. We thus recommend to use the single-relay scheme with soft-decoding strategy. For very low $\triangle S N R(\leq 8 \mathrm{~dB})$, the multi-relay cooperation with soft decoding outperforms the other schemes. With 6 relays, the gain compared to the single-relay scheme with soft decoding increases as $\triangle S N R$ decreases $(3.5 \mathrm{~dB}$ at $\Delta S N R=2 \mathrm{~dB})$. A high BER at relay output is the bottleneck of the cooperation scheme. We can reduce it by increasing the test pattern number used in the relay soft decoding Chase algorithm, and thus improve the performance of single-relay solution. If this improvement is not sufficient, we can increase the relays with respect to the system constraints (energy, data rate, etc.).

\section{CONCLUSION}

In this paper, we have studied the impact of relay-generated errors on the performance of the cooperative scheme proposed in [12]. The relay-generated error correlation yields an error floor at the destination. To reduce this phenomenon, we have proposed to use multiple relays. When associated to a pseudo-random selection of the redundancy sent to the destination, the scheme is efficient, all the more as the correlation degree is high, which is the case of poor source-relay channel conditions. For a fast fading channel with medium to high source-relay channel SNR, the single-relay cooperation scheme achieves the best trade-off between bit error rate and complexity. For very poor source-relay channel conditions, the relay soft-decoding performance can be improved by adding test patterns. If not sufficient, multiple relays can be used with respect to the system requirements.

Future work will focus on the diversity gain brought by multi-relays on a block fading channel and the analysis from an information theory viewpoint of this cooperative scheme.

\section{REFERENCES}

[1] G. Kramer and A.J. van Wijngaarden, "On the White Gaussian MultipleAccess Relay Channel," In Proc. IEEE Int. Symp. on Inform. Theory (ISIT), pp. 40, June 2000.

[2] L. Sankaranarayanan, G. Kramer and N.B. Mandayam, "Hierarchical sensor networks: capacity bounds and cooperative strategies using the multiple-access relay channel model," in Proc. 42nd Ann. Allerton Conf. Commun., Control, Computing, pp. 191-199, Sept. 2004.

[3] C. Hausl and P. Dupraz, "Joint Network-Channel Coding for the MultipleAccess Relay Channel," 3rd Annual IEEE Commun. Society on Sensor and Ad Hoc Commun. and Networks (SECON), pp. 817-812, Sept. 2006.

[4] T.M. Cover and A.E. Gamal, "Capacity theorems for the relay channel," IEEE Trans. Info. Theory, vol. 25, no. 5, pp. 572-84, Sept. 1979.

[5] A. Nosratinia, T.E. Hunter and A. Hedayat, "Cooperative communication in wireless networks," IEEE Commun. Magazine, vol. 42, no. 10, pp. 74-80, Oct. 2004

[6] R. Ahlswede, N. Cai, S.-Y.R. Li and R.W. Yeung, "Network information flow," IEEE Trans. on Inform. Theory, pp. 1204-1216, July 2000.

[7] R. Koetter and M. Médard, "An algebraic approach to network coding," IEEE Trans. Networking, vol. 11, no. 5, pp. 782-795, Oct. 2003.

[8] R. Pyndiah, "Near-optimum decoding of product codes: block turbo codes," IEEE trans. Commun., vol. 46, no. 8, pp. 1003-1010, Aug. 1998.

[9] Z. Xia, Y. Qu, H. Yu and Y. Xu, "A distributed cooperative product code for multi-source single destination wireless network," in Proc. 15th AsiaPacific Conf. on Commun. (APCC), pp. 736-739, Oct. 2009.

[10] T.u.R. Ahsin and S.B. Slimane, "Network coding based on product codes in cooperative relaying," IEEE Wireless Commun. and Networking Conf. (WCNC), pp. 1-6, April 2010.

[11] E.A. Obiedat, G. Chen and L. Cao, "Distributed turbo product codes over multiple relays," IEEE 7th Consumer Commun. and Networking Conf. (CCNC), pp. 1-5, Jan. 2010.

[12] R. Pyndiah, F. Guilloud and K. Amis, "Multiple source cooperative coding using turbo product codes with a noisy relay," in Proc. 6th Inter. Symp. on Turbo Code \& Iterative Inform.(ISTC), pp. 98-102, Sept. 2010.

[13] J.G. Proakis and M. Salehi, "Digital communications," Mc Graw Hill Press, fifth edition, 2008.

[14] D. Chase, "A class of algorithms for decoding block codes with channel measurement information," IEEE Trans. Inform. Theory, vol.IT-18, no.1, Jan. 1972. 